\title{
Particle Physics from the Asian Perspective
}

\author{
Yuji Yamazaki* \\ Kobe University \\ E-mail: yamazaki@phys.sci.kobe-u.ac.jp
}

An overview on the past and present experiments in high-energy physics and future plan on collider physics and other major programmes in Asian region are presented. The experiments in Asia emerged mainly from projects on flavour physics, while the new projects in China and Japan includes also the energy-frontier accelerators. The current status of present and future projects is reported.

XXIII International Workshop on Deep-Inelastic Scattering 27 April - May 12015

Dallas, Texas

${ }^{*}$ Speaker. 


\section{Introduction}

The oral presentation corresponding to this proceedings document was to provide an overall picture on present high-energy physics (HEP) in Asian area, together with future direction. Asia has no strongly-united body like European Committee for Future Accelerators (ECFA) in Europe, nor a country with strong initiative like US for north America, although the Asian HEP communities are tied and exchanging information through Asian Committee for Future Accelerators (ACFA). There is no common strategy among Asian countries. Instead, statuses of the current and future projects from each country were investigated from available material and summarised in the talk. Some comments were made as a personal view.

This document is organised as follows. First, an overview on the past and present experiments of HEP in Asia are given and compared with those of North America and Europe. Then, the current and near-future projects in high-energy physics in Asia, mostly in China, Korea and Japan, are reviewed. Finally, the present statuses of the two major future plans in Asia, the Circular Electron Positron Collider (CEPC) in China and the International Linear Collider (ILC) in Japan, are given.

Since this review is a collection of snapshots for each project and the information becomes quickly obsolete, the description in this document is limited short, and instead references to the original information are given.

This contribution does not cover the programs related to HEP not using accelerators, such as underground experiments for dark matter searches, neutrino-less double-beta decays, and gravitational wave experiments and cosmic-frontier observatories on surface and in space, where Asian funding sources are involved.

\section{HEP programs in Asia and other regions: past and present}

The first large-scale HEP programs in Asia were TRISTAN, a general-purpose $e^{+} e^{-}$colliders in KEK, Japan, and the Kamiokande water Čerenkov experiment for observing proton decays as well as various sources of neutrinos, including that from the SuperNova SN 1978a [1]. The evidence of neutrino oscillations observed by the Super-Kamiokande observatory in 1998a [2] has lead to many experiments measuring parameters of neutrino oscillations. In Asia, such examples are KamLAND for medium-baseline reactor experiments; T2K for the long-baseline accelerator experiments; and the reactor-neutrino experiments, namely Daya Bay in China and RENO in Korea. Meanwhile, the TRISTAN ring was transformed to KEKB accelerator as a B-factory, on which the Belle experiment has experimentally proven [3] the validity of the Kobayashi-Maskawa theory, together with the BaBar experiment in SLAC, US, in 2001.

Looking back these past and present experiments, one would notice that there is no energyfrontier experiment in Asia except for the TRISTAN accelerator, which was the highest energy $e^{+} e^{-}$collider in the world for some period but rather quickly taken over by the LEP experiment at CERN. Figure 2 1 shows a schematic diagram of the HEP experiments with accelerators and neutrino observatories, with horizontal axis taken as an approximate energy scale, vertical axis as "flavourness", which indicates how large fraction of the experiment addresses the question of mysteries in lepton or quark flavours such as neutrino oscillation, charged lepton-flavour violation, $\mathrm{CP}$-violation and new physics through rare decays of heavy quarks. The area of the circles represents the size 
of the experiments. One finds that many of the large circles on the right side, i.e. energy-frontier experiments, are either European or North American projects, while Asia nowadays dominates in flavour physics.

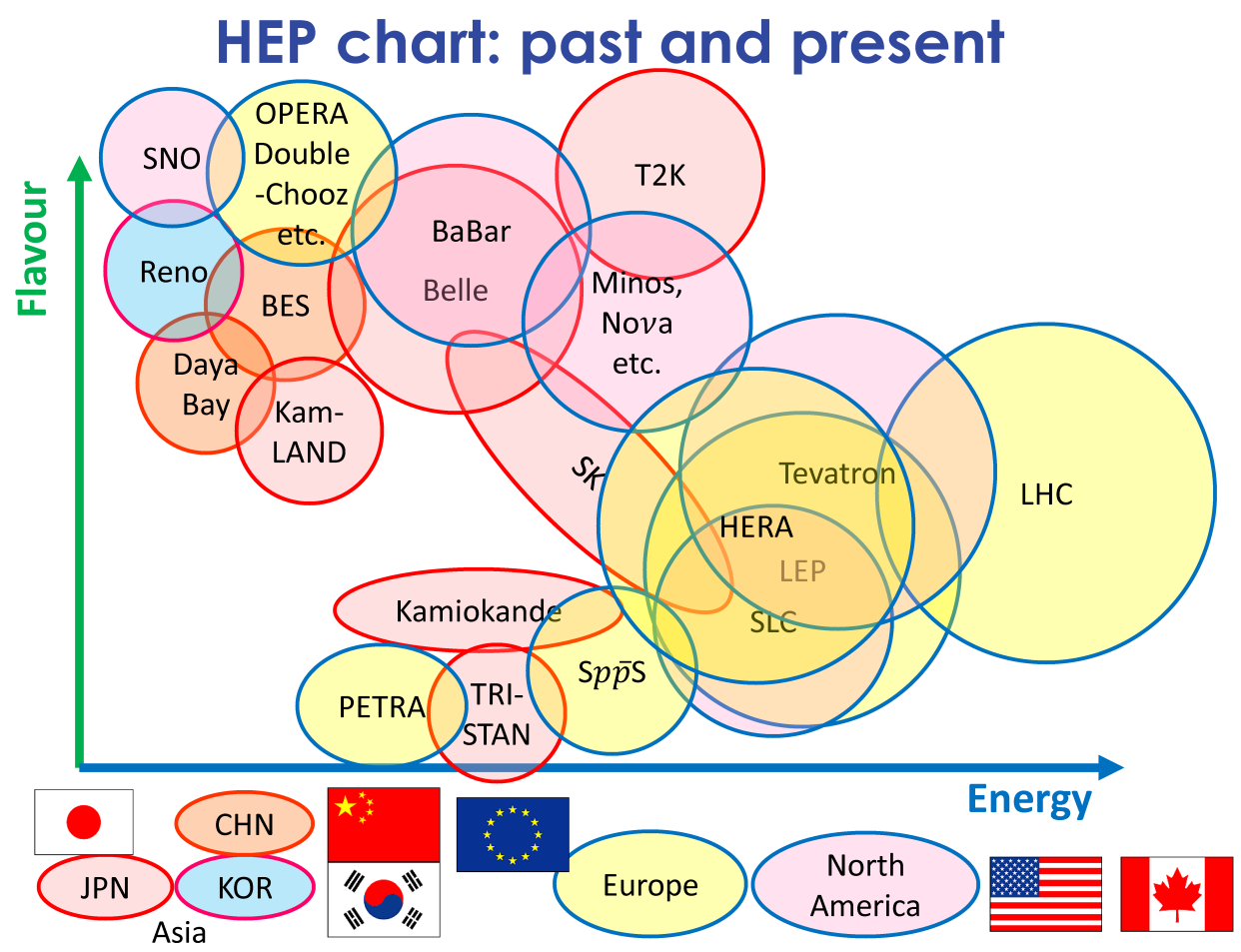

Figure 1: A schematic chart of the past and present HEP experiments. The vertical axis indicates how large fraction the experiments addresses the question on flavour physics. The area of the circles represents the size of the experiments.

\section{Future experiments for flavour physics in Asia}

The flavour physics continues to be a major part of HEP experimental program in Asia. Many experiments are either under construction or in late R\&D stages.

For quark flavour sector, the largest-scale experiment is the SuperKEKB accelerator and the Belle II experiment. The first pilot run is planned in 2017 without the vertex detector. Full operation is expected from 2018. The Belle and Belle II experiment has been playing an important role for internationalisation of the Japanese HEP program. The number of authors from Japan is just around $20 \%$ of total researchers of more than 600 people participating the experiment. The main collaborators indeed come from Asia-Pacific region ( $>40 \%)$; Korea, Taiwan, India, Australia and China have more than ten participants.

The COMET experiment is being under construction at J-Parc. The experiment searches for charged lepton-flavour violation through $\mu \rightarrow e$ conversion. Again a large number of participants are from Asia, including China, Korea, India, Saudi Arabia, Malaysia and Vietnam. 
In the neutrino sector, a next generation of the water-Čerenkov-based neutrino detector, the Hyper-Kamiokande (Hyper-K) is being designed. The volume of the water is 25 times higher than the Super-Kamiokande, increasing the number of neutrino events injected from J-Parc by a factor of 50, thanks also to increased intensity of the accelerator. Hyper-K has been attracting a large number of people in Europe and some from US, while more active participation from Asia-Pacific are being pursued: so far Korea is the only country from Asia.

Since Hyper-K is a very large project, requiring funding at the order of a billion USD, the proposal of the project has been already examined through several committees, leading to positive recommendations so far. It was originally proposed through the Japan Association of High-Energy Physics (JAHEP), gave a recommendation [4] in 2012 that the Hyper-K project is one of the two high-priority projects together with the ILC. It was then submitted to the Science Council of Japan (SCJ), when it called for large-scale project proposals of various scientific societies, including social science, medical science and technologies. The Hyper-K project was selected as a toppriority project by the SCJ in "Master Plan 2014" report [5] of large research projects. The MEXT (the Ministry of Education, Culture, Sports, Science and Technology), however, did not include the Hyper-K project in their "Roadmap 2014" [6, which is the list of the projects to be investigated for final approval. The next roadmap will be issued in 2017. Should it be successful, the earliest construction may start as early as 2018 and start to have operation in 2025.

Another big project in Asia for the neutrino physics is Jiangmen Underground Neutrino Oscillation experiment (JUNO), a medium-distance reactor-based oscillation experiment. The detector will be located at a distance of about $60 \mathrm{~km}$ to observe the maximum deficit of the neutrino oscillation. The experiment is also sensitive to the mass hierarchy. The site construction is already started and will be completed in 2017. The data taking will start in 2020 .

\section{QCD in Asia}

Many of the Asian countries participate in out-of-area experiments such as LHC collider experiments, ATLAS and CMS, as well as heavy-ion experiments such as STAR, PHENIX and Alice experiments. This includes also countries like Iran and Indonesia, which has not yet participating to Asian experiments. The QCD physics is another driving force for increasing Asian participation to the HEP world.

\section{Future circular colliders in China}

The next-generation project in China is the CEPC, which will be followed by the SppC collider using the same tunnel like the LEP and the LHC. The circumference of the ring is $50-70 \mathrm{~km}$. The energy for the $e^{+} e^{-}$option is from 90 to $250 \mathrm{GeV}$, enough for producing the discovered Higgs boson through the Higgs-Strahlung; the machine serves as a Higgs factory for precision measurements of the Higgs properties. The energy for $p p$ collisions are above $50 \mathrm{TeV}$, far exceeding the LHC energy and will be a discovery machine as well for the SM precision test via triple and quartic coupling of the Higgs boson.

The preliminary Conceptual Design Report (preCDR) for this project has been released in March 2015. Earliest possible start time for construction of the CEPC collider is 2021, with its 
physics run starting at 2028. The SppC construction and the physics run follows that. The big advantage of this project is that the it is well conceivable to construct a large facility in the current situation of China [7].

\section{The ILC and its status}

The ILC project has submitted the technical design report in 2013. The selected construction site in Japan is Tohoku Kitakami area, which was chosen for scientific reasons. The site allows to extend its energy reach up to $\sqrt{s}=1 \mathrm{TeV}$. Its main physics targets are precision determination of Higgs couplings, including $t \bar{t} H$ and $h^{3}$ couplings, wider search for SUSY particles and top precision measurements.

The ILC project is under examination through various governmental review processes. As described above, the ILC project is one of top two priority projects for Japanese HEP community. In response to the 2013 statement of JAHEP, KEK has submitted a proposal of the ILC to SCJ in 2013. The ILC project was not listed on the master plan of SCJ because of its scale in budget beyond the scope of SCJ. Instead, a separated report [8] was issued. The report requests MEXT to study the following items:

1. Physics research strategy of ILC in the view of LHC upgrade path

2. A funding scheme not affecting other activities in science and other national projects

3. Domestic organisation to implement the project

4. Human resources for construction, operation and, in particular, leadership positions

Receiving the report, MEXT initiated the Academic Expert Committee, below which a few working groups were formed. They are in charge of investigating some of the above-listed questions, consisting of the physics working group (for question 1) and the TDR validation working group. As of May 2015, another working group on human resources (for question 4) is also being formed. As for the question 3, KEK has recently established the ILC planning office. The working groups should come to its conclusion not later than 2016 and should give a report ${ }^{1}$.

The question 2, the funding scheme, necessarily involves international cost sharing. This is an issue beyond MEXT, which requires government-to-government discussions on issues and preparation for that. In parallel, there are movements by policy makers to support the project, such as adopting resolutions, giving policy reports to the Japanese prime minister as well as visiting US diet members, first in 2014 and also in May 2015. Similar visits to to European and Asian countries are planned in 2015 winter.

The fact that MEXT and diet members talk to the governments of other countries before taking any decision may not be easily understandable from outside. The reason is that they need to know how other countries view the project before they proceed; they need feedbacks. The Japanese government has to make sure the reaction of other countries are positive when taking a decision. There is no way to proceed with this project without support from outside the country.

\footnotetext{
${ }^{1}$ The report has became public after this talk: it was issued on 25 June 2015 and can be found in English under: http://www.mext.go.jp/component/b_menu/shingi/toushin/_icsFiles/afieldfile/2015/08/05/1360596_3.pdf
} 
The necessity of international cooperation is not only for the cost but also for human resources. Since such sharing cannot be avoided, it will, by construction, become a true large international project. The ILC can be the first trial of such kind in Asia. This is exactly the point where the Japanese governments and politicians are being motivated [9].

\section{Summary}

As shown schematically, large-scale energy-frontier experiments in high-energy physics are yet to be realised in Asia, while the contribution to the flavour physics is already significant. People in Asian area, not only HEP and accelerator physicists but also politicians, strongly wishes to contribute to the community by having future colliders in Asia.

\section{Acknowledgement}

I would like to thank to all of the people listed below for providing material as well as information on the situation: Prof. Yifang Wang (IHEP) for China and IHEP; Prof. Tsuyoshi Nakaya (Kyoto U.) and Prof. Masahi Yokoyama (U. Tokyo) for Hyper-K; Prof. Yutaka Ushiroda for BelleII; Prof. Kenta Shigaki for PHENIX and Alice; and Prof. Toshinori Mori and Akimasa Ishikawa for the ILC.

\section{References}

[1] K. Hirata et al. (Kamiokande-II Collaboration), Observation of a Neutrino Burst from the Supernova SN 1987a, PRL 58 (1987) 1490.

[2] Y. Fukuda et al. (Super-Kamiokande Collaboration), Evidence for Oscillation of Atmospheric Neutrinos, PRL 81 (1998) 1562.

[3] K. Abe et al. (Belle Collaboration), Observation of large CP violation in the neutral B meson system, PRL 87 (2001) 091802.

[4] Japan Association of High Energy Physicists, The Final Report of the Subcommittee on Future Projects of High Energy Physics, http://www.jahep.org/office/doc/201202_hecsubc_report.pdf .

[5] Science Council of Japan, Japanese Master Plan of Large Research Projects 2014 (in Japanese), http://www.scj.go.jp/ja/info/kohyo/pdf/kohyo-22-t188-1.pdf .

[6] Ministry of Education, Culture, Sports, Science and Technology (MEXT), Roadmap 2014 (in Japanese), http://www.mext.go.jp/b_menu/shingi/gijyutu/gijyutu4/toushin/_icsFiles/afieldfile/2014/08/26/1351171_1.pdf .

[7] For example, Yifang Wang (IHEP), IHEP status and future plan, Asian Linear Collider Workshop (ALCW2015), 23 April 2015, KEK, Tsukuba, Japan, https://agenda.linearcollider.org/event/6557/session/13/contribution/16/material/slides/0.ppt .

[8] Science Council of Japan, Report on the International Linear Collider Project, http://www.scj.go.jp/ja/info/kohyo/pdf/Report\%20on\%20ILC_Exective\%20Summary.pdf .

[9] T. Mori, Closing remarks, Asian Linear Collider Workshop (ALCW2015), 23 April 2015, KEK, Tsukuba, Japan, https://agenda.linearcollider.org/event/6557/session/13/contribution/20/material/slides/0.pdf . 